UCLA $/ 92 / \mathrm{TEP} / 41$

\title{
Explicit Calculation of the Renormalized Singlet Axial Anomaly
}

\author{
Michiel Bos* \\ Department of Physics \\ University of California \\ Los Angeles, CA 90024
}

\begin{abstract}
A careful and complete discussion is given of the renormalization of the singlet axial anomaly equation in a vector-like nonabelian gauge theory such as QCD regularized by ordinary dimensional regularization. Pseudotensorial structures are treated with the 't Hooft-Veltman prescription. A general framework for calculations is developed, and subsequently verified by explicit computations through two loops. This is followed by a discussion of the matrix elements obtained.
\end{abstract}

$11 / 92$

* e-mail: bos@uclahep.jnet, or bos@uclahep.physics.ucla.edu. 


\section{Introduction}

The axial anomaly [1,2, 3] is by now a standard feature of gauge field theory. In spite of this the study of its renormalization has still not reached a state of stable equilibrium. The literature on the question, which began more than twenty years ago with the classical work of Adler and Bardeen [4], has grown both by the emergence of new issues and by the reconsideration of old ones, a process that continues to the present day. Particularly notable revivals of interest have been spurred by the paradox of the currents in supersymmetric theories (see e.g. [5] for an early formulation of the problem) and, more recently, by new developments concerning the spin of the proton (reviewed e.g. in [6]).

The present paper intends to contribute to the topic an explicit perturbative computation at the two-loop level for a vector-like nonabelian gauge theory such as QCD. More precisely, I will calculate the two-gluon matrix elements of all the operators in the singlet anomaly equation to second nontrivial order in dimensionally regularized perturbation theory, and apply the results to an analysis of the equation itself. The choice of ordinary dimensional regularization (as against the dimensional reduction of much of the supersymmetric literature, e.g. [7]) is based on its prominence in perturbative studies of nonabelian gauge theories, as well as its relatively obvious consistency. The prescription of 't Hooft and Veltman [8] is adopted for the pseudotensorial objects $\gamma_{5}$ and $\epsilon^{\mu \nu \rho \sigma}$. This type of approach is of course not novel in itself; the present investigation is similar in spirit to several earlier ones, notably [9] and [10]. I have gone beyond this earlier work by performing calculations that are both more comprehensive and more complete; I have also gone to greater lengths in connecting the actual computations to their conceptual underpinnings.

The first two sections following this introduction are devoted to a general derivation of the renormalized anomaly equation. First I review the operators of interest, normalizing them with reference to their scaling properties to the extent allowed by universality; renormalization group invariance of a renormalized anomaly equation then leads to strong a posteriori constraints on its coefficients. This line of argument, which originated in early work on the intertwining of the anomaly and Callan-Symanzik equations [11,12], has by now become rather commonplace 
in the literature, where one finds it expounded with varying degrees of precision. Next I adopt a constructive approach, regularizing the theory via dimensional regularization, deriving the anomaly equation and renormalizing it, and matching the result with the scheme-independent template obtained before.

Section 4, which is the core of the paper, contains most of the actual calculations performed. Apart from the very validity of the renormalized anomaly equation they verify the finite renormalizations required in the formalism of dimensional regularization. In view of the role of renormalization group arguments in organizing the problem I have employed the background field method, within which the renormalization of the nonabelian theory is most analogous to that of the well-understood abelian case. I pay particular attention to the normalization of the gluon tensor, which has been taken for granted in previous treatments along similar lines.

The final section is devoted to comments on the matrix elements obtained, mostly from points of view suggested by the abelian Adler-Bardeen theorem [4]. The discussion eventually touches on an ongoing discussion about a possible renormalization of the vacuum angle in QCD [13,14,15].

The literature on anomalies is so extensive as to preclude exhaustive citation. My list of references is a selection of the papers best known to me, and I apologize to authors of omitted work.

The system considered consists of a generically nonabelian gauge field $A$ with coupling constant $g^{2}$, and fermions $\psi$ of mass $m$ forming $n_{R}$ copies of an irreducible representation $R$ of the gauge group. The group invariants $c_{R}$ and $T_{R}$ are defined in the usual manner, $\operatorname{tr} T^{a} T^{b}=T_{R} \delta_{a b}, T^{a} T^{a}=c_{R} \mathbf{1}$, where the $T^{a}$ are the group generators, $\left[T^{a}, T^{b}\right]=i f_{a b c} T^{c}$; in particular, for the adjoint representation $c_{A} \delta_{a b}=$ $f_{a c d} f_{b c d}$. I denote the dimensionality of the regulating spacetime of dimensional regularization by $d$ and write $d=4-\epsilon$.

\section{Operator Normalization}

This section reviews the general structure of the anomaly equation. The theory is assumed to be regularized in some gauge-invariant way, but the details of the regularization will be irrelevant. The point is to describe a sensible normalization of relevant operators by concentrating on scaling behavior and universality arguments. 
The following auxiliary consideration will be required. Suppose one is given a renormalized (finite) operator $\mathcal{O}$; suppose moreover that its renormalization was multiplicative and its anomalous scaling therefore of the form

$$
\mu \frac{d}{d \mu} \mathcal{O}=-\gamma_{\mathcal{O}}\left(g^{2}\right) \mathcal{O}
$$

where $g^{2}$ is the coupling constant and $\mu$ is a renormalization mass. (I will write all expressions in such a way that they are naturally interpreted in dimensional regularization with minimal or quasiminimal subtraction by taking $\mu$ to be the familiar scale factor of the extra dimensions, but no firm commitment to that procedure is necessary.) A finite multiplicative renormalization of $\mathcal{O}$ is a replacement of $\mathcal{O}$ by $\mathcal{O}^{\prime} \equiv f\left(g^{2}\right) \mathcal{O}, f\left(g^{2}\right)$ being a power series in $g^{2}$ with $f(0)=1$; the latter restriction excludes redefinitions of the classical operator corresponding to $\mathcal{O}$ that have nothing to do with the ambiguities of renormalized perturbation theory. The anomalous dimension of $\mathcal{O}^{\prime}$ differs in general from that of $\mathcal{O}$ and is given by

$$
\gamma_{\mathcal{O}^{\prime}}\left(g^{2}\right)=\gamma_{\mathcal{O}}\left(g^{2}\right)-\beta\left(g^{2}\right) \frac{f^{\prime}\left(g^{2}\right)}{f\left(g^{2}\right)} .
$$

The power series for the beta-function starts at order $g^{4}$ and that of $\gamma_{\mathcal{O}}$ typically at order $g^{2}$, but if $\gamma_{\mathcal{O}}$ has no one-loop term, $\gamma_{\mathcal{O}}=c g^{4}+\ldots$, the quotient $\gamma_{\mathcal{O}} / \beta$ is a power series in $g^{2}$ and a finite renormalization $f$ may be found such that the renormalized operator $\mathcal{O}^{\prime}$ has vanishing anomalous dimension,

$$
f\left(g^{2}\right)=\exp \left[\int_{0}^{g^{2}} d x \frac{\gamma_{\mathcal{O}}(x)}{\beta(x)}\right] .
$$

On the other hand, the part of the anomalous dimension that arises at one loop is unaffected by finite renormalizations, hence irremovable. Similar reasoning applies to additive renormalizations; terms of order $g^{4}$ and beyond in the power series expansion of an off-diagonal anomalous dimension are removable by a finite renormalization, while the one-loop, $g^{2}$ term is universal.

Anomalous Ward identities for the singlet axial current relate insertions in Green functions of three operators: the divergence of $j_{5}^{\mu}$, the singlet axial current itself; $j_{5}$, the pseudoscalar density; and the anomalous term $F \tilde{F}$. In addition they 
involve contact terms corresponding to axial transformations of the fermion fields. To lowest nontrivial order one finds the familiar expression

$$
\partial_{\mu} j_{5}^{\mu}=2 m j_{5}+\frac{n_{R} T_{R} g^{2}}{16 \pi^{2}} \epsilon^{\mu \nu \rho \sigma} F_{\mu \nu}^{a} F_{\rho \sigma}^{a}+\text { c.t. }
$$

"c.t." being the contact terms. Upon insertion in a Green function of fermion fields $\psi\left(x_{1}\right), \ldots, \psi\left(x_{p}\right), \bar{\psi}\left(y_{1}\right), \ldots, \bar{\psi}\left(y_{q}\right)$ and gauge fields $A\left(z_{1}\right), \ldots, A\left(z_{r}\right)$, "c.t." takes the form

$$
\begin{aligned}
& -\sum_{i=1}^{p} \delta\left(w-x_{i}\right)<\psi\left(x_{1}\right) \ldots \gamma_{5} \psi\left(x_{i}\right) \ldots \psi\left(x_{p}\right) \bar{\psi}\left(y_{1}\right) \ldots \bar{\psi}\left(y_{q}\right) A\left(z_{1}\right) \ldots A\left(z_{r}\right)> \\
& \quad-\sum_{j=1}^{q} \delta\left(w-y_{j}\right)<\psi\left(x_{1}\right) \ldots \psi\left(x_{p}\right) \bar{\psi}\left(y_{1}\right) \ldots \bar{\psi}\left(y_{j}\right) \gamma_{5} \ldots \bar{\psi}\left(y_{q}\right) A\left(z_{1}\right) \ldots A\left(z_{r}\right)>
\end{aligned}
$$

involving the axial transformations of the fermions.

Classically the singlet axial current equals $\bar{\psi} \gamma^{\mu} \gamma_{5} \psi$; its quantum version is renormalized multiplicatively. The one-loop diagram contributing to that (Figure 1) is identical to the diagram for the vector current $\bar{\psi} \gamma^{\mu} \psi$ but for an extra $\gamma_{5}$ at the vertex. The inclusion of this $\gamma_{5}$ does not spoil the familiar cancellation of infinities between the one-loop correction and the wave function renormalization of the constituent fermion fields, so $j_{5}^{\mu}$, too, is finite at one loop and has no one-loop anomalous dimension. By the argument reviewed above one infers the existence of a (unique) normalization that makes the scaling of the axial current canonical to all orders; call this current $\left[j_{5}^{\mu}\right]_{C}$. We may and will take the renormalized divergence of the axial current to be the divergence of the renormalized axial current.

Similar reasoning applies to the pseudoscalar density $j_{5}$, classically equal to $i \bar{\psi} \gamma_{5} \psi$. Its multiplicative renormalization involves at one loop a diagram as in Figure 1. Now an analogy obtains with the one-loop renormalization of $\bar{\psi} \psi$, the renormalization constant of which is the inverse of that of the mass. The combination $m j_{5}$ is thus found to scale canonically at one loop and may be taken, via a finite renormalization of $j_{5}$ if necessary, to have that property to all orders. I denote the resulting object by $m\left[j_{5}\right]_{C}$.

The composite operator $F \tilde{F} \equiv \epsilon^{\mu \nu \rho \sigma} F_{\mu \nu}^{a} F_{\rho \sigma}^{a}$ is the derivative of the gaugevariant Chern-Simons current $4 \epsilon^{\mu \nu \rho \sigma}\left(A_{\nu}^{a} \partial_{\rho} A_{\sigma}^{a}+\frac{1}{3} f_{a b c} A_{\nu}^{a} A_{\rho}^{b} A_{\sigma}^{c}\right)$. The renormalization of this current is not exhausted by a multiplicative factor, but involves mixing 
with the axial fermion current as well. (By contrast, the axial current itself is protected by its gauge invariance from non-diagonal renormalization involving the gauge-variant Chern-Simons current.) Correspondingly $F \tilde{F}$ mixes with $\partial_{\mu} j_{5}^{\mu}$.

Figures 2 and 3 contain one-loop diagrams relevant for the diagonal and offdiagonal renormalization of $F \tilde{F}$, respectively. The analysis of Figure 2 is simplified if background field Feynman rules are used, and I will do so from now on. The logarithmically divergent parts of the diagrams in Figure 2 then cancel among each other, so the diagonal, multiplicative scaling behavior of $F \tilde{F}$ is derived entirely (at least to this order) from the wave function renormalizations of its constituent gluons. In the background field formalism the renormalization of the gluon field is by construction exactly the inverse of that of the coupling constant, so the product $g^{2} F \tilde{F}$ is not renormalized and free of anomalous dimension at one loop, again as far as diagonal renormalization is concerned. The option of a finite renormalization of $F \tilde{F}$ may then be invoked to extend this property to all orders. A renormalized version of $F \tilde{F}$ will be denoted by $[F \tilde{F}]_{C}$ if its product with $g^{2}$ has canonical diagonal scaling.

This leaves the issue of additive renormalization and off-diagonal scaling, to be parametrized by

$$
\mu \frac{d}{d \mu}\left(g^{2}[F \tilde{F}]_{C}\right)=-\gamma_{F 5}\left(g^{2}\right) g^{2} \partial_{\mu}\left[j_{5}^{\mu}\right]_{C} .
$$

The extra $g^{2}$ on the right hand side balances the one on the left in order that the counting of powers of $g^{2}$ in the anomalous dimension $\gamma_{F 5}$ be identical to that of the number of loops at which they arise. Evaluation of the diagram in Figure 3 shows $\gamma_{F 5}$ to be nonvanishing at one loop; the logarithmically divergent part of that diagram is of the form

$$
-\frac{3 i c_{R} g^{2}}{4 \pi^{2}} \ln \frac{\Lambda^{2}}{\mu^{2}}(\not p '-\not p) \gamma_{5}
$$

in momentum space, $\Lambda$ being a cutoff and $\mu$ a renormalization scale, and $p$ and $p^{\prime}$ the momenta of the fermions. This implies a one-loop value $\gamma_{F 5}=3 c_{R} g^{2} / 2 \pi^{2}$. Higher orders may be changed by additive renormalizations of $[F \tilde{F}]_{C},\left([F \tilde{F}]_{C}\right)^{\prime} \equiv$ $[F \tilde{F}]_{C}+f\left(g^{2}\right) \partial_{\mu}\left[j_{5}^{\mu}\right]_{C}$ with $f(0)=0$, but there is no particularly natural way of fixing this freedom and I will not commit myself with regard to it, leaving the ambiguity of $[F \tilde{F}]_{C}$ implicit in the notation. 
(There exists another approach to the normalization of $g^{2} F \tilde{F}$, ultimately equivalent to the present one, in which the Chern-Simons current is related by gauge or BRS descent to a naturally finite operator, the normalization of which fixes the diagonal normalization of the renormalized $g^{2} F \tilde{F}$; see [16, 17, 18].)

Now assume that the anomaly equation survives renormalization, in the sense that there exists in the complete theory a relation of linear dependence involving the renormalized operators defined above and reducing to (2.4) at the lowest nontrivial order in perturbation theory. Write this relation in the form

$$
C_{1}\left(g^{2}\right) \partial_{\mu}\left[j_{5}^{\mu}\right]_{C}=C_{2}\left(g^{2}\right) 2 m\left[j_{5}\right]_{C}+C_{3}\left(g^{2}\right) \frac{n_{R} T_{R}}{16 \pi^{2}} g^{2}[F \tilde{F}]_{C}+c . t . .
$$

where the $C_{i}$ are power series in $g^{2}$, with $C_{i}(0)=1$ to accommodate (2.4), and the overall factor has been fixed by normalizing the contact terms to their standard value, 2.5. Application of the renormalization group operator $\mu \frac{d}{d \mu}$ to both sides yields

$$
\beta C_{1}^{\prime} \partial_{\mu}\left[j_{5}^{\mu}\right]_{C}=\beta C_{2}^{\prime} 2 m\left[j_{5}\right]_{C}+\beta C_{3}^{\prime} \frac{n_{R} T_{R}}{16 \pi^{2}} g^{2}[F \tilde{F}]_{C}-C_{3} \frac{n_{R} T_{R}}{16 \pi^{2}} g^{2} \gamma_{F 5} \partial_{\mu}\left[j_{5}^{\mu}\right]_{C}
$$

The contact terms have disappeared because they are finite as they stand, see (2.5), hence without anomalous dimension. Without them the remaining operators are independent and consistency requires

$$
C_{2}^{\prime}=C_{3}^{\prime}=0, \quad C_{1}^{\prime}=-\frac{n_{R} T_{R} g^{2} \gamma_{F 5}}{16 \pi^{2} \beta} C_{3},
$$

i.e.

$$
C_{1}=1-\frac{n_{R} T_{R}}{16 \pi^{2}} \int_{0}^{g^{2}} d x \frac{x \gamma_{F 5}(x)}{\beta(x)}, \quad C_{2}=C_{3}=1
$$

The single remaining non-universal feature (at least by the criteria espoused in the above) may be hidden in notation by introducing a finite renormalization of the canonical axial current,

$$
\left[j_{5}^{\mu}\right]_{A B} \equiv C_{1}\left(g^{2}\right)\left[j_{5}^{\mu}\right]_{C},
$$

in terms of which the renormalized anomaly equation reads

$$
\partial_{\mu}\left[j_{5}^{\mu}\right]_{A B}=2 m\left[j_{5}\right]_{C}+\frac{n_{R} T_{R}}{16 \pi^{2}} g^{2}[F \tilde{F}]_{C}+c . t .
$$

In keeping with the (mostly supersymmetric) literature $\left[j_{5}^{\mu}\right]_{A B}$ may be called an Adler-Bardeen current, with indefinite article to signal the normalization ambiguity which it inherits, via $C_{1}$, from the mixing dimension $\gamma_{F 5}$. Along comes a multiplicative anomalous dimension starting at order $g^{4}$ 4,19. 


\section{Dimensional Regularization}

The next step is to derive the anomaly in the framework of dimensional regularization. This discussion is complementary to the previous one in concentrating on aspects that are peculiar to a specific regularization scheme. For convenience I will mostly adhere to the conventions and notation of Collins' textbook on renormalization [20], where the Ward identity for the (non-anomalous) non-singlet current is derived.

To define the axial current in the regulated theory requires a definition for $\gamma_{5}$, which is notoriously problematic in dimensional regularization. In the 't HooftVeltman prescription [8,21] $\gamma_{5}$ is taken as the product of the first four $\gamma$-matrices at the cost of anticommutativity with all $\gamma$ 's in $d$ dimensions. This requires a distinction between the first four dimensions and the remaining $(d-4)$, to which end one introduces projectors denoted by a superscripted bar and hat, respectively; a vector $a^{\mu}$ is written as $\bar{a}^{\mu}+\hat{a}^{\mu}$, with $\bar{a}^{\mu}=a^{\mu}$ if $\mu=0,1,2,3$ and 0 otherwise, and the other way around for $\hat{a}^{\mu}$. One may then proceed to write down identities for tensorial objects with contracted indices. The totally antisymmetric tensor $\epsilon_{\mu \nu \rho \sigma}$ is introduced as a bar-carrying object in the above sense, i.e. $\epsilon_{\mu \nu \rho \sigma}$ equals its usual value when each index is $0,1,2$, or 3 , and 0 otherwise; for brevity of notation the bar will not be indicated explicitly. Finally, $\gamma_{5}$ is defined by

$$
\gamma_{5}=\frac{i}{4 !} \epsilon_{\mu \nu \rho \sigma} \gamma^{\mu} \gamma^{\nu} \gamma^{\rho} \gamma^{\sigma}
$$

Its most notable feature is its failure to anticommute with the hatted $\gamma$ 's:

$$
\left\{\gamma^{5}, \bar{\gamma}^{\mu}\right\}=0 \text { but }\left[\gamma^{5}, \hat{\gamma}^{\mu}\right]=0
$$

Trace identities may be established as usual: the trace of $\gamma_{5}$ times a product of $n$ $\gamma$-matrices vanishes if $n$ is odd or less than four, while (taking $\operatorname{tr} \mathbf{1}=4$ )

$$
\operatorname{tr} \gamma_{5} \gamma^{\mu} \gamma^{\nu} \gamma^{\rho} \gamma^{\sigma}=4 i \epsilon^{\mu \nu \rho \sigma}
$$

The bare singlet axial current and pseudoscalar density in the dimensionally regulated theory are now defined by

$$
j_{5}^{\mu} \equiv \bar{\psi}_{0} \bar{\gamma}^{\mu} \gamma_{5} \psi_{0}
$$




$$
j_{5} \equiv i \bar{\psi}_{0} \gamma_{5} \psi_{0}
$$

$\psi_{0} \equiv\left(Z_{2}\right)^{\frac{1}{2}} \psi$ is the bare fermion wave function. The bar on $\gamma^{\mu}$ in the axial current, which does not affect the classical limit, is necessary for hermiticity.

The would-be conservation law for the axial current is the unrenormalized anomaly equation,

$$
\partial_{\mu} j_{5}^{\mu}=2 m_{0} j_{5}+\mathcal{O}_{a n}+\mathcal{O}_{c t}
$$

where $\mathcal{O}_{a n}$ and $\mathcal{O}_{c t}$ are given by

$$
\mathcal{O}_{a n} \equiv \frac{1}{2} \bar{\psi}_{0}\left\{\overleftrightarrow{\not D}, \gamma_{5}\right\} \psi_{0}
$$

and

$$
\mathcal{O}_{c t} \equiv i \bar{\psi}_{0} \gamma_{5}\left(i \not D-m_{0}\right) \psi_{0}-i \bar{\psi}_{0}\left(i \overleftarrow{\not D}+m_{0}\right) \gamma_{5} \psi_{0}
$$

The operator $\mathcal{O}_{a n}$ represents the potential anomaly. Only the hatted components of the covariant derivation occur; $\mathcal{O}_{a n}$ is "evanescent" 20 and would vanish if no divergences occurred in the theory in the limit $d \rightarrow 4$. $\mathcal{O}_{c t}$ is proportional to the equation of motion and vanishes on shell, but its insertion in covariant Green functions, where derivatives and time-ordering are taken to commute, produces the contact terms associated with axial transformations of the fermion as given explicitly by (2.5).

The next step is to renormalize the equation, for which we adopt minimal subtraction. The linearity of that procedure implies that the subtracted equation is obtained by replacing each operator by its minimally subtracted version (and $m_{0}$ by the minimally renormalized mass $m$; we assume the coupling $g^{2}$ and wave function renormalizations such as $Z_{2}$ to be defined by minimal subtraction as well). The minimally subtracted current $\left[j_{5}^{\mu}\right]_{M S}$ and pseudoscalar density $\left[j_{5}\right]_{M S}$ are both multiplicatively related to the respective unrenormalized operators. The contact terms are manifestly finite, $\left[\mathcal{O}_{c t}\right]_{M S}=\mathcal{O}_{c t}$. These relations and the anomaly equation implicitly determine how the minimally subtracted anomaly operator $\left[\mathcal{O}_{a n}\right]_{M S}$ is to be defined in terms of unrenormalized operators and renormalization factors.

The crucial point is now that in the limit $d \rightarrow 4$ finite contributions involving the subtracted anomaly term can only arise in diagrams where the evanescent vertex on which that term is built multiplies an internal divergence. As a result, 
$\left[\mathcal{O}_{a n}\right]_{M S}$ survives in the limit $d \rightarrow 4$ as a linear combination of local operators with non-evanescent basic vertices, the net effect being a reduction in the number of independent operators. The coefficients of this linear combination are (at least in perturbation theory) power series in $g^{2}$ without constant terms, because $\left[\mathcal{O}_{a n}\right]_{M S}$ vanishes at the tree level. Other examples of this phenomenon have been worked out in contexts rather different from the present one [22,23].

The set of non-evanescent operators in terms of which $\left[\mathcal{O}_{a n}\right]_{M S}$ is expressed may be restricted by dimensional and symmetry considerations. That leaves $\partial_{\mu}\left[j_{5}^{\mu}\right]_{M S}$, $m\left[j_{5}\right]_{M S}, \mathcal{O}_{c t}$ and $[F \tilde{F}]_{M S}$, the latter being the minimally subtracted version of the composite operator $F \tilde{F} \equiv \epsilon^{\mu \nu \rho \sigma}\left(F_{\mu \nu}^{a}\right)_{0}\left(F_{\rho \sigma}^{a}\right)_{0} . \quad\left(\left(F_{\mu \nu}^{a}\right)_{0} \equiv\left(Z_{3}\right)^{\frac{1}{2}}\left(F_{\mu \nu}^{a}\right)_{0}\right.$ is the bare field strength, simply proportional to the renormalized one in the background field method.) $\mathcal{O}_{c t}$ may be replaced by other operators by use of the minimally subtracted anomaly equation and the remainder may be rearranged as

$$
\left[\mathcal{O}_{a n}\right]_{M S}=f_{1}\left(g^{2}\right) \partial_{\mu}\left[j_{5}^{\mu}\right]_{M S}+f_{2}\left(g^{2}\right) m\left[j_{5}\right]_{M S}+f_{3}\left(g^{2}\right)[F \tilde{F}]_{M S}
$$

where the $f_{i}$ are all finite and of order $g^{2}$ at least. Substitution in the minimally subtracted anomaly equation yields

$$
\left(1-f_{1}\left(g^{2}\right)\right) \partial_{\mu}\left[j_{5}^{\mu}\right]_{M S}=\left(1+\frac{1}{2} f_{2}\left(g^{2}\right)\right) 2 m\left[j_{5}\right]_{M S}+f_{3}\left(g^{2}\right)[F \tilde{F}]_{M S}+\mathcal{O}_{c t} .
$$

The existence of the anomaly thus amounts to $f_{3} \neq 0$.

The factors $1-f_{1}$ and $1+\frac{1}{2} f_{2}$ may be removed by a finite renormalization, leading to oversubtracted (with respect to minimal subtraction, that is) operators:

$$
\begin{gathered}
{\left[j_{5}^{\mu}\right]_{O S} \equiv\left(1-f_{1}\left(g^{2}\right)\right)\left[j_{5}^{\mu}\right]_{M S}} \\
{\left[j_{5}\right]_{O S} \equiv\left(1+\frac{1}{2} f_{2}\left(g^{2}\right)\right)\left[j_{5}\right]_{M S} .}
\end{gathered}
$$

An analogous oversubtraction may be performed on $F \tilde{F} ; f_{3}$, if nonzero, has the form $f_{3}^{(1)} g^{2}+\mathcal{O}\left(g^{4}\right)$ with $f_{3}^{(1)} \neq 0$, so $f_{3}\left(g^{2}\right) /\left(f_{3}^{(1)} g^{2}\right)$ is a power series starting with 1 and I renormalize

$$
[F \tilde{F}]_{O S} \equiv \frac{f_{3}\left(g^{2}\right)}{f_{3}^{(1)} g^{2}}[F \tilde{F}]_{M S}
$$

leading to a rewritten renormalized anomaly equation

$$
\partial_{\mu}\left[j_{5}^{\mu}\right]_{O S}=2 m\left[j_{5}\right]_{O S}+f_{3}^{(1)} g^{2}[F \tilde{F}]_{O S}+\mathcal{O}_{c t} .
$$


This brings the construction to the point where contact is made with the discussion of the previous section. This time the renormalized anomaly equation has been derived, rather than assumed. Comparison with (2.13) identifies $\left[j_{5}^{\mu}\right]_{O S}$ as an Adler-Bardeen current and $m\left[j_{5}\right]_{O S}$ as the canonically scaling pseudoscalar density $m\left[j_{5}\right]_{C} ; g^{2}[F \tilde{F}]_{O S}$ is a product of the type $g^{2}[F \tilde{F}]_{C}$ with canonical diagonal scaling behavior. The value of the off-diagonal anomalous dimension of $g^{2}[F \tilde{F}]_{O S}$ beyond one loop as well as the related anomalous dimension of $\left[j_{5}^{\mu}\right]_{O S}$ emerge as specifics of the regularization.

\section{Calculations}

In this section I describe the calculation of quantities relevant to the next-toleading order two-gluon matrix element of the anomaly equation.

The kinematical setting is as follows. We will compute one-particle irreducible functions of two gauge fields with a single operator insertion, that operator being one of those that occur in the anomaly equation. The labeling of the external gauge fields will be as in Figure 4 , with $(a, b)$ and $(\mu, \nu)$ group and Lorentz indices, respectively, and $p$ and $-p^{\prime}$ the incoming gauge field momenta. For the operators of interest $\mathcal{O}$ the two-gluon function has the form

$$
\epsilon_{\mu \nu \rho \sigma} p^{\rho} p^{\prime \sigma} \delta_{a b} \hat{\Gamma}_{\mathcal{O}}\left(p, p^{\prime} ; m\right)
$$

where $\hat{\Gamma}_{\mathcal{O}}$, to be referred to as the reduced matrix element, is a function of the scalars $p^{2}, p^{2}$ and $p \cdot p^{\prime}$ as well as of the fermion mass $m$. Where an expression for $\hat{\Gamma}_{\mathcal{O}}$ for all $p$ and $p^{\prime}$ is not practicable or illuminating I will specialize to the infrared regime $p^{\prime} \rightarrow p$, accessible from the complete function after differentiation with respect to $p$, say. I will then also eliminate either $p^{2}$ or $m^{2}$; in each case the renormalized reduced matrix element, classically dimensionless, will depend on the remaining parameter at most via renormalization group logarithms. All computations are performed using the background field Feynman rules, a convenient table (and motivation) of which may be found in [24].

The following ingredients are required for an evaluation of the two-gluon matrix element of the anomaly equation (3.14) through next-to-leading order. The matrix 
elements of $\partial_{\mu}\left[j_{5}^{\mu}\right]_{M S}$ and $m\left[j_{5}\right]_{M S}$, which have their lowest nonzero contributions at one loop, should be calculated at one and two loops. The matrix element of $[F \tilde{F}]_{M S}$ starts at the tree level and we need the one-loop contribution in addition to that. The functions $f_{1}\left(g^{2}\right)$ and $f_{2}\left(g^{2}\right)$ contribute to the anomaly equation from the next-to-leading level onward and their leading (i.e. $g^{2}$ ) terms suffice. Of the function $f_{3}\left(g^{2}\right)$, on the other hand, both the $g^{2}$ and the $g^{4}$ terms are required. The two-gluon matrix element of $\mathcal{O}_{c t}$ is zero.

We begin with the reduced matrix elements $\hat{\Gamma}_{\partial_{\mu}\left[j_{5}^{\mu}\right]_{M S}}$ and $\hat{\Gamma}_{m\left[j_{5}\right]_{M S}}$. The lowest order contributions stem from the familiar triangle graph, Figure 4, and are easily computed for general $p$ and $p^{\prime}$. The results have been well-known since the early investigations of the anomaly,

$$
\begin{gathered}
\left.\hat{\Gamma}_{\partial_{\mu}\left[j_{5}^{\mu}\right]_{M S}}\left(p, p^{\prime} ; m\right)\right|_{\text {one loop }}=-\frac{g^{2} n_{R} T_{R}}{\pi^{2}}\left(\frac{1}{2}+m^{2} I_{00}\left(p, p^{\prime} ; m\right)\right) \\
\left.\hat{\Gamma}_{m\left[j_{5}\right]_{M S}}\left(p, p^{\prime} ; m\right)\right|_{\text {one loop }}=-\frac{g^{2} n_{R} T_{R}}{2 \pi^{2}} m^{2} I_{00}\left(p, p^{\prime} ; m\right)
\end{gathered}
$$

where

$$
I_{00}\left(p, p^{\prime} ; m\right) \equiv \int_{0}^{1} d s \int_{0}^{1-s} d t \frac{1}{p^{2} t(1-t)+p^{2} s(1-s)-2 p \cdot p^{\prime} s t-m^{2}} .
$$

If the fermion mass vanishes,

$$
\left.\hat{\Gamma}_{\partial_{\mu}\left[j_{5}^{\mu}\right]_{M S}}\left(p, p^{\prime} ; m=0\right)\right|_{\text {one loop }}=-\frac{g^{2} n_{R} T_{R}}{2 \pi^{2}}, \quad \hat{\Gamma}_{m\left[j_{5}\right]_{M S}}\left(p, p^{\prime} ; m=0\right)=0
$$

by contrast, if the fermion mass is finite but $p^{\prime}=p, p^{2} \rightarrow 0$

$$
\begin{gathered}
\left.\hat{\Gamma}_{\partial_{\mu}\left[j_{5}^{\mu}\right]_{M S}}\left(p=p^{\prime}, p^{2}=0 ; m\right)\right|_{\text {one loop }}=0 \\
\left.\hat{\Gamma}_{m\left[j_{5}\right]_{M S}}\left(p=p^{\prime}, p^{2}=0 ; m\right)\right|_{\text {one loop }}=\frac{g^{2} n_{R} T_{R}}{4 \pi^{2}} .
\end{gathered}
$$

We proceed to the contributions of order $g^{4}$. The relation between minimally subtracted and unrenormalized reduced matrix elements is

$$
\hat{\Gamma}_{[\mathcal{O}]_{M S}}=Z_{\mathcal{O}} Z_{A} \hat{\Gamma}_{\mathcal{O}, \text { bare }}
$$

with $Z_{\mathcal{O}}$ the renormalization constant of $\mathcal{O}$ as determined by minimal subtraction, $\mathcal{O}$ being any of the operators of interest; $Z_{A}^{\frac{1}{2}}$ is the wave function renormalization 
of the gauge field. For arbitrary $p, p^{\prime}$ and $m$ the expressions for the reduced matrix elements are rather unwieldy and I concentrate on the aforementioned limits.

At the two-loop level $\hat{\Gamma}_{\partial_{\mu} j_{5}^{\mu}, \text { bare }}$ and $\hat{\Gamma}_{j_{5}, \text { bare }}$ involve the eleven diagrams of Figure 5. The values of these diagrams with (1) $\mathcal{O}=\partial_{\mu} j_{5}^{\mu}, p=p^{\prime}, p^{2} \neq 0, m=0,(2)$ $\mathcal{O}=\partial_{\mu} j_{5}^{\mu}, p=p^{\prime}, p^{2} \rightarrow 0, m \neq 0$, and $(3) \mathcal{O}=j_{5}, p=p^{\prime}, p^{2} \rightarrow 0, m \neq 0$, are listed in Tables 1, 2 and 3, respectively. They have been computed using the background field Feynman rules. As we only determine terms through order $g^{4}$ of the left hand side of (4.7), the bare parameters $g_{0}^{2}$ and $m_{0}$ have been replaced in the tables by the lowest order of their renormalized expansions, $g^{2} \mu^{4-d}$ and $m$. A common factor $g^{4} n_{R} T_{R} /(4 \pi)^{4}$ has been omitted; $L_{m}$ stands for $\ln \frac{m^{2}}{4 \pi \mu^{2}}+\gamma$ and $L_{p}$ for $\ln \frac{-p^{2}}{4 \pi \mu^{2}}+\gamma$. The gauge dependence of the gluon Feynman rules becomes an issue as one finds terms up to quadratic order in the standard covariant gauge parameter $\alpha$.

Consider first $\partial_{\mu} j_{5}^{\mu}$ for massless fermions. The two-loop diagrams of Table 1 add up to

$$
\begin{aligned}
\hat{\Gamma}_{\partial_{\mu} j_{5}^{\mu}, \text { bare }}( & \left.=p^{\prime}, p^{2} \neq 0 ; m=0\right)\left.\right|_{\text {two loops }} \sim \\
- & \frac{g^{4} n_{R} T_{R}}{8 \pi^{4}}\left(c_{R}+c_{A}\left(1+\frac{3}{4}(1-\alpha)-\frac{1}{8}(1-\alpha)^{2}\right)\right) .
\end{aligned}
$$

The similarity sign is meant to express the replacement of bare parameters by their lowest order renormalized ones. The absence of a term proportional to $c_{R} \alpha$ corresponds to the gauge invariance of the result for QED and obtains equally from the other two tables. It is not hard to show by diagrammatic reasoning that such terms must cancel independently of the values of $p, p^{\prime}$ or $m$. It may also be noted that the propagator-induced corrections to the triangle, diagrams I through III, vanish at $\alpha=0$, a consequence of the vanishing of the one-loop self-energy of massless fermions in the Landau gauge. Proceeding to the counterterms, the bare one-loop matrix element is $-\frac{g_{0}^{2} n_{R} T_{R}}{2 \pi^{2}}\left(p^{2}\right)^{(d-4) / 2}$, as (4.5) with the regulating dimensionality $d$ taken into account; but the bare coupling in this expression is converted to the finite renormalized one by multiplication with $Z_{A}$ as in (4.7), because the renormalization of the wave function is precisely the inverse of that of the coupling in the background field method. As for the renormalization factor $Z_{\partial_{\mu} j_{5}^{\mu}}$, it is equal to 1 at one loop by the calculation sketched in section 2 . The renormalized reduced matrix element 
becomes, through two loops,

$$
\begin{aligned}
\hat{\Gamma}_{\partial_{\mu}\left[j_{5}^{\mu}\right]_{M S}}(p & \left.=p^{\prime}, p^{2} \neq 0 ; m=0\right)=-\frac{g^{2} n_{R} T_{R}}{2 \pi^{2}} \\
& -\frac{g^{4} n_{R} T_{R}}{8 \pi^{4}}\left(c_{R}+c_{A}\left(1+\frac{3}{4}(1-\alpha)-\frac{1}{8}(1-\alpha)^{2}\right)\right) .
\end{aligned}
$$

If the fermions are taken to be massive the two-loop unrenormalized matrix element of $\partial_{\mu} j_{5}^{\mu}$ is zero in the limit $p=p^{\prime}, p^{2} \rightarrow 0$, as is found by adding the entries of Table 2; as a result the vanishing of the renormalized matrix element persists to order $g^{4}$,

$$
\hat{\Gamma}_{\partial_{\mu}\left[j_{5}^{\mu}\right]_{M S}}\left(p=p^{\prime}, p^{2} \rightarrow 0 ; m \neq 0\right)=0
$$

This extends to the nonabelian case the corresponding property of the divergence of the axial current in massive QED, proven to all orders by Adler and Bardeen [4]; we shall return to this presently.

Turning to the pseudoscalar density $j_{5}$, which occurs in the anomaly equation multiplied by $m$ and is therefore relevant only in the case of massive fermions, the contributions to the limit $p=p^{\prime}, p^{2} \rightarrow 0$ tabulated in Table 3 add up to

$$
\begin{aligned}
& \left.\hat{\Gamma}_{j_{5}, \text { bare }}\left(p=p^{\prime}, p^{2} \rightarrow 0 ; m \neq 0\right)\right|_{\text {two loops }} \sim \\
& \frac{g^{4} n_{R} T_{R}}{16 \pi^{4} m}\left(\frac{1}{2} c_{R}+c_{A}\left(1+\frac{3}{4}(1-\alpha)-\frac{1}{8}(1-\alpha)^{2}\right)\right) .
\end{aligned}
$$

Note that though the infrared behavior of individual diagrams produces terms involving logarithms of $p^{2}$, such terms cancel in the sum. The bare one-loop result is here

$$
\left.\hat{\Gamma}_{j_{5}, \text { bare }}\left(p=p^{\prime}, p^{2} \rightarrow 0 ; m \neq 0\right)\right|_{\text {one loop }}=\frac{g_{0}^{2} n_{R} T_{R}}{4 \pi^{2} m_{0}}\left(m_{0}\right)^{d-4} .
$$

The counterterms implicit in $g_{0}^{2}$ cancel against those generated by $Z_{A}$, as before. A similar cancellation arises between $\frac{1}{m_{0}}$ and $Z_{j_{5}}$; we saw in section 2 that the oneloop renormalization of $j_{5}$ is opposite to that of $m, m_{0} j_{5}=m\left[j_{5}\right]_{M S}$. That leaves only the scale factor, which by use of the familiar minimal subtraction for the mass

$$
m_{0}=\left(1-\frac{3 g^{2} c_{R}}{8 \pi^{2} \epsilon}+\ldots\right) m
$$


is found to contribute $3 g^{4} c_{R} n_{R} T_{R} / 32 \pi^{4} m$ to the reduced matrix element. We obtain, to two loops,

$$
\begin{aligned}
\hat{\Gamma}_{\left[j_{5}\right]_{M S}}(p & \left.=p^{\prime}, p^{2} \rightarrow 0 ; m \neq 0\right)=\frac{g^{2} n_{R} T_{R}}{4 \pi^{2} m} \\
& +\frac{g^{4} n_{R} T_{R}}{16 \pi^{4} m}\left(2 c_{R}+c_{A}\left(1+\frac{3}{4}(1-\alpha)-\frac{1}{8}(1-\alpha)^{2}\right)\right) .
\end{aligned}
$$

Thus $\hat{\Gamma}_{2 m\left[j_{5}\right]_{M S}}$ for massive fermions is similar, but not completely identical, to $\hat{\Gamma}_{\partial_{\mu}\left[j_{5}^{\mu}\right]_{M S}}$ for massless ones.

The results above may also be arrived at by subtracting subdivergences diagram by diagram. This allows for the special case of the Feynman gauge comparison with some of the ordinary perturbation theory results of [10], which are presented in this form; I have found agreement wherever I checked. Upon further restriction to massless fermions one also finds agreement with the older results of [9]. Restriction both to massless fermions and to the group structure $c_{R}$ produces a value given in [25].

The next task is to find the functions $f_{1}\left(g^{2}\right)$ and $f_{2}\left(g^{2}\right)$ at order $g^{2}$. This may be done by computing the one-loop matrix element of the anomalous operator $\mathcal{O}_{a n}$ with a fermion and a conjugate fermion field; the tree graph vanishes in the limit $d \rightarrow 4$. The relevant one-loop diagrams are shown in Figure 6. After inclusion of the wave function renormalization of the constituent fermions of $\mathcal{O}_{a n}$ one finds the finite result, in a general gauge $\alpha$,

$$
\frac{i g^{2} c_{R}}{4 \pi^{2}}\left(\overline{\not p}^{\prime}-\overline{\not p}\right) \gamma_{5}-\frac{i g^{2} c_{R}}{\pi^{2}} m \gamma_{5} .
$$

This is rewritten in the form of the right hand side of (3.9) by comparison with the basic vertices of $\partial_{\mu} j_{5}^{\mu}$ and $j_{5}$, equal to $i\left(\overline{p h}^{\prime}-\overline{\not p}\right) \gamma_{5}$ and $i \gamma_{5}$, respectively, and observing that the $\psi-\bar{\psi}$ matrix element of $F \tilde{F}$ starts only at order $g^{2}$. It follows that

$$
f_{1}\left(g^{2}\right)=\frac{g^{2} c_{R}}{4 \pi^{2}}+\ldots, \quad f_{2}\left(g^{2}\right)=-\frac{g^{2} c_{R}}{\pi^{2}}+\ldots
$$

Obviously this is basically an abelian effect, hence independent of $\alpha$.

The above results may now be combined into two-loop expressions for the reduced matrix elements of the oversubtracted fermionic operators $\partial_{\mu}\left[j_{5}^{\mu}\right]_{O S} \equiv(1-$ 
$\left.f_{1}\right) \partial_{\mu}\left[j_{5}^{\mu}\right]_{M S}$ and $\left[j_{5}\right]_{O S} \equiv\left(1+\frac{1}{2} f_{2}\right)\left[j_{5}\right]_{M S}$ in a general covariant gauge $\alpha$ :

$$
\begin{gathered}
\hat{\Gamma}_{\partial_{\mu}\left[j_{5}^{\mu}\right]_{O S}}\left(p=p^{\prime}, p^{2} \neq 0 ; m=0\right)=-\frac{g^{2} n_{R} T_{R}}{2 \pi^{2}} \\
-\frac{g^{4} c_{A} n_{R} T_{R}}{8 \pi^{4}}\left(1+\frac{3}{4}(1-\alpha)-\frac{1}{8}(1-\alpha)^{2}\right) \\
\hat{\Gamma}_{\partial_{\mu}\left[j_{5}^{\mu}\right]_{O S}}\left(p=p^{\prime}, p^{2} \rightarrow 0 ; m \neq 0\right)=0 . \\
\hat{\Gamma}_{m\left[j_{5}\right]_{O S}}\left(p=p^{\prime}, p^{2} \rightarrow 0 ; m \neq 0\right)=\frac{g^{2} n_{R} T_{R}}{4 \pi^{2}} \\
+\frac{g^{4} c_{A} n_{R} T_{R}}{16 \pi^{4}}\left(1+\frac{3}{4}(1-\alpha)-\frac{1}{8}(1-\alpha)^{2}\right)
\end{gathered}
$$

The finite renormalization has had the effect of eliminating terms involving the quadratic Casimir of the fermion representation from the $g^{4}$ contributions.

We now turn to the matrix element of $[F \tilde{F}]_{M S}$. The tree level value, determined by the basic vertex of $F \tilde{F}$, is $\left.\hat{\Gamma}_{[F \tilde{F}]_{M S}}\right|_{\text {tree }}=-8$. The next order is obtained from a formula of the form (4.7). The unsubtracted one-loop diagrams are shown in Figure 2 ; in accordance with the considerations of section 2, their sum is finite if $g_{0}^{2}$ is replaced by $g^{2} \mu^{4-d}$. The renormalization constant $Z_{F \tilde{F}}$ must therefore be chosen to compensate for the wave function renormalizations of the gluon fields in $F \tilde{F}$, $Z_{F \tilde{F}}=\left(Z_{A}\right)^{-1}$. With this choice, $\hat{\Gamma}_{[F \tilde{F}]_{M S}}$ at one loop is found to be, for arbitrary $p, p^{\prime}$,

$$
\begin{gathered}
\left.\hat{\Gamma}_{[F \tilde{F}]_{M S}}\right|_{\text {one loop }}=-\frac{2 g^{2} c_{A}}{\pi^{2}}\left\{1-\frac{1}{2}\left(p-p^{\prime}\right)^{2} I_{00}\left(p, p^{\prime} ; 0\right)\right. \\
+\frac{1}{4}(1-\alpha)\left[1+\int_{0}^{1} d s \int_{0}^{1-s} d t \frac{1-s-t}{\Delta}\left((1-2 s) p^{2}+(1-2 t) p^{2}+2(s+t) p \cdot p^{\prime}\right)\right. \\
\left.\left.+\left(p-p^{\prime}\right)^{2} \int_{0}^{1} d s \int_{0}^{1-s} d t \frac{1-s-t}{\Delta^{2}}\left(t(1-s) p^{2}+s(1-t) p^{2}\right)\right)\right] \\
\left.-\frac{1}{8}(1-\alpha)^{2}\right\}
\end{gathered}
$$

with $I_{00}$ as in (4.4) and

$$
\Delta \equiv p^{2} t(1-t)+p^{2} s(1-s)-2 p \cdot p^{\prime} s t
$$


This somewhat unsavory expression has an easily read off limit as $p^{\prime} \rightarrow p$; including tree and one-loop effects,

$$
\hat{\Gamma}_{[F \tilde{F}]_{M S}}\left(p^{\prime}=p\right)=-8\left(1+\frac{g^{2} c_{A}}{4 \pi^{2}}\left(1+\frac{3}{4}(1-\alpha)-\frac{1}{8}(1-\alpha)^{2}\right)\right) .
$$

Note that to this order the fermions play no role in $\hat{\Gamma}_{[F \tilde{F}]_{M S}}$.

The one-loop matrix element of $F \tilde{F}$ was computed using a different intermediate regularization in [26]. There the term proportional to $(1-\alpha)$ is presented as

$$
-\frac{c_{A} g^{2}}{\pi^{2}}(1-\alpha)\left[1-\frac{1}{4}\left(p-p^{\prime}\right)^{2}\left(p^{2} \frac{\partial}{\partial p^{2}}+p^{2} \frac{\partial}{\partial p^{\prime 2}}\right) I_{00}\right]
$$

where the partial derivatives are defined by treating $I_{00}\left(p, p^{\prime} ; 0\right)$ as a function of the invariants $p^{2}, p^{\prime 2}$ and $p \cdot p^{\prime}$. This appears to yield $\frac{1}{2}(1-\alpha)$ instead of $\frac{3}{4}(1-\alpha)$ in (4.22) [15], but $I_{00}$ is singular as $p^{\prime} \rightarrow p$,

$$
\begin{aligned}
& I_{00}\left(p, p^{\prime} ; 0\right) \rightarrow-\frac{4}{\left(p+p^{\prime}\right)^{2}} \ln \frac{\left(p^{\prime}-p\right)^{2}}{\left(p^{\prime}+p\right)^{2}} \\
& \left(p^{2} \frac{\partial}{\partial p^{2}}+p^{\prime 2} \frac{\partial}{\partial p^{\prime 2}}\right) I_{00} \rightarrow-\frac{2}{\left(p^{\prime}-p\right)^{2}}
\end{aligned}
$$

and this eliminates the apparent discrepancy (cf. also [14]).

With an eye on (3.14) we turn now to the determination of $f_{3}^{(1)}$, the Taylor coefficient of $g^{2}$ in $f_{3}\left(g^{2}\right)$. As with $f_{1}$ and $f_{2}$, the $g^{2}$ term in $f_{3}$ may be extracted from a one-loop diagram, in this case the triangle with two gluons (Figure 4). With $\mathcal{O}_{a n}$ at the upper vertex this graph yields, in the limit $d \rightarrow 4$ (to eliminate evanescent contributions) 8

$$
\left.\hat{\Gamma}_{\mathcal{O}_{a n}}\left(p, p^{\prime} ; m\right)\right|_{\text {one loop }}=-\frac{g^{2} n_{R} T_{R}}{2 \pi^{2}}
$$

This is compatible with (3.9) provided

$$
f_{3}\left(g^{2}\right)=\frac{g^{2} n_{R} T_{R}}{16 \pi^{2}}+\ldots
$$

as the first two terms on the right hand side of 3.9 contribute to $\hat{\Gamma}_{\mathcal{O}_{a n}}$ only at the $g^{4}$ level. $f_{3}^{(1)}$ is thus determined to be $n_{R} T_{R} / 16 \pi^{2}$, and one recognizes in it the well-known coefficient of the anomaly. 
We may now verify this by evaluating the two-gluon matrix element of the renormalized anomaly equation (3.14) using the results obtained above. There are no contact terms. The reduced matrix element of $\partial_{\mu}\left[j_{5}^{\mu}\right]_{O S}-2 m\left[j_{5}\right]_{O S}$ was found to be the same in the two kinematical settings studied through two loops,

$$
\begin{aligned}
\hat{\Gamma}_{\partial_{\mu}\left[j_{5}^{\mu}\right]_{O S}}-2 \hat{\Gamma}_{m\left[j_{5}\right]_{O S}} & =-\frac{g^{2} n_{R} T_{R}}{2 \pi^{2}} \\
& -\frac{g^{4} c_{A} n_{R} T_{R}}{8 \pi^{4}}\left(1+\frac{3}{4}(1-\alpha)-\frac{1}{8}(1-\alpha)^{2}\right) .
\end{aligned}
$$

But this is exactly equal to $f_{3}^{(1)} \hat{\Gamma}_{[F \tilde{F}]_{M S}}$ in the limit $p^{\prime} \rightarrow p$, so the validity of the renormalized anomaly equation requires that there be no oversubtraction for $F \tilde{F}$, i.e., that $[F \tilde{F}]_{M S}$ and $[F \tilde{F}]_{O S}$ be the same object.

The verification of this consistency requirement, which previous treatments [9, 10] have omitted altogether, occupies the remainder of this section. One way of accomplishing it would be to directly compute the term of order $g^{4}$ in $f_{3}$ that gives the finite renormalization relating $[F \tilde{F}]_{M S}$ to $[F \tilde{F}]_{O S}$ at second order. I will forego this option for a less direct and therefore maybe more interesting check. $[F \tilde{F}]_{O S}$ is characterized among the finite multiplicative renormalizations of $[F \tilde{F}]_{M S}$ by the property that $g^{2}[F \tilde{F}]_{O S}$ has no anomalous diagonal scaling. Ergo, $[F \tilde{F}]_{M S}$ is equal to $[F \tilde{F}]_{O S}$ if and only if its diagonal anomalous dimension is exactly $\beta\left(g^{2}\right) / g^{2}$.

For this to be the case the minimal subtraction constant $Z_{F \tilde{F}}$ must be equal to $Z_{A}^{-1}$ through two loops; we already know that to be true at one loop. In view of (4.7) this amounts to $\hat{\Gamma}_{F \tilde{F}, \text { bare }}$ being finite if its parameters are expanded to order $g^{4}$.

The thirty-six two-loop diagrams contributing to $\hat{\Gamma}_{F \tilde{F} \text {, bare }}$ are shown in Figure 7. Diagrams in which both external gluons couple to the same four-point vertex may be discarded immediately by symmetry considerations and have been omitted; no orientations are indicated on fermion and ghost lines and it is understood that both choices are to be considered. Table 4 lists the pole parts of these diagrams in the Feynman gauge $(\alpha=1)$ as computed for $p^{\prime} \rightarrow p$; for the diagrams involving fermions, the limits $p^{2} / m^{2} \rightarrow 0$ and $m^{2} / p^{2} \rightarrow 0$ are distinguished if necessary. Each contribution of the form $\frac{1}{\epsilon^{2}}$ is to be supplemented by a $-\frac{1}{\epsilon} L_{p}$ term of equal strength, left out of the table for the sake of brevity. 
The contributions of order $g^{4}$ carry three distinct group theoretical structures, namely $c_{A}^{2}, c_{A} n_{R} T_{R}$, and $c_{R} n_{R} T_{R}$. The cancellation of infinities we are after should obtain for each structure separately.

Consider first the $c_{A}^{2}$ terms. Retaining only those terms is tantamount to assuming that the fermions are absent from the theory, which reduces the set of diagrams to numbers I through XXIX. The sum of these diagrams as per Table 4 is

$$
\left.\hat{\Gamma}_{F \tilde{F}, \text { bare }}\right|_{\text {two loops }} \sim-\frac{59 c_{A}^{2} g^{4}}{48 \pi^{4} \epsilon} .
$$

The cancellation of $\frac{1}{\epsilon^{2}}$ poles in this sum, with the accompanying cancellation of terms of the form $\frac{1}{\epsilon} L_{p}$, reflects the absence of divergences in the one-loop matrix element [27]. The counterterms follow from expanding $g_{0}^{2}$ and the bare gauge pa-

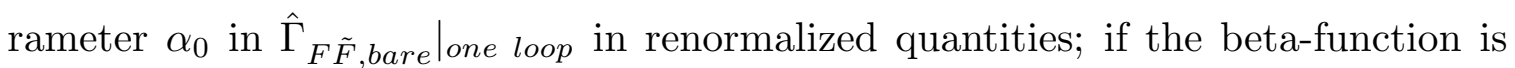
written as $b_{1} g^{4}+\ldots$ and the renormalization group coefficient of the gauge parameter, $\mu \frac{d}{d \mu} \alpha$, as $d_{1} g^{2}+\ldots$, that amounts to applying

$$
\frac{b_{1} g^{4}}{\epsilon} \frac{\partial}{\partial g^{2}}+\frac{d_{1} g^{2}}{\epsilon} \frac{\partial}{\partial \alpha}
$$

to the expression in (4.22). With the well-known values $b_{1}=-11 c_{A} / 24 \pi^{2}$ and $d_{1}=5 c_{A} \alpha / 24 \pi^{2}$ that leads in the Feynman gauge to a counterterm contribution exactly opposite to (4.28). This shows that the $g^{4}$ part of $\hat{\Gamma}_{F F, \text { bare }}$ is finite and hence to this order in pure QCD $Z_{F \tilde{F}}=Z_{A}^{-1}$, as desired.

Upon re-inclusion of the fermions there are seven more two-loop diagrams, each involving a single fermion loop, shown as numbers XXX through XXXVI in Figure 7. The Feynman gauge pole parts of these diagrams, again computed at $p^{\prime}=p$, are sensitive to the value of $p^{2} / \mathrm{m}^{2}$. Adding up the relevant entries of Table 4 produces

$$
\left.\delta \hat{\Gamma}_{F \tilde{F}, \text { bare }}\right|_{\text {two loops }} \sim \frac{7 g^{4} c_{A} n_{R} T_{R}}{12 \pi^{4} \epsilon}+ \begin{cases}\frac{3 g^{4} c_{R} n_{R} T_{R}}{4 \pi^{4} \epsilon} & \text { if } m^{2} / p^{2} \rightarrow 0 \\ 0 & \text { if } p^{2} / m^{2} \rightarrow 0 .\end{cases}
$$

Concomitantly the values of $b_{1}$ and $d_{1}$ receive the well-known fermionic contributions $\delta b_{1}=n_{R} T_{R} / 6 \pi^{2}$ and $\delta d_{1}=-n_{R} T_{R} \alpha / 6 \pi^{2}$. It is easy to check that the pole terms with group structure $c_{A} T_{R}$ cancel between the new diagrams and the $(\alpha=1)$ 
counterterm modifications. Thus it only remains to account for the $c_{R} n_{R} T_{R}$ part of 4.30 ).

Inspection of Table 4 shows that the net $c_{R} n_{R} T_{R}$ contribution arises from diagram XXXI. The upper part of that diagram is identical to the one-loop mixing diagram of $F \tilde{F}$ and $\partial_{\mu} j_{5}^{\mu}$, Figure 3. In fact it is not $F \tilde{F}$ but $F \tilde{F}+\frac{3 c_{R} g^{2}}{2 \pi^{2} \epsilon} \partial_{\mu} j_{5}^{\mu}$ that is multiplicatively related to $[F \tilde{F}]_{M S}$, where the pole is that of the mixing diagram; this formula accounts for (2.7), with $\ln \frac{\Lambda}{\mu}$ replaced by $\frac{1}{\epsilon}$. The renormalization formula for $F \tilde{F}$, (4.7), should be modified accordingly. In view of the one-loop values of $\hat{\Gamma}_{\partial_{\mu} j_{5}^{\mu}}\left(p^{\prime}=p\right)$ in the two limiting regimes of $p^{2} / m^{2},(4.5-6)$, this effect provides a correction that precisely cancels the $c_{R} n_{R} T_{R}$-dependent term in (4.30). The peculiar dependence of (4.30) on the presence or absence of a fermion mass is thus seen to be inherited from the analogous feature of the divergence of the axial current.

This concludes the consistency check (at least as far as the Feynman gauge is concerned): through two loops the minimally subtracted operator $[F \tilde{F}]_{M S}$ scales as the inverse of the minimally subtracted coupling constant, without the need for a finite renormalization.

\section{Comments}

In the previous section the two-gluon matrix elements of a number of suitably normalized operators were computed through two loops in order to verify the validity of the renormalized anomaly equation. I will now consider those matrix elements in their own right.

The first issue that should be addressed is the nature of the various kinematical limits. Most of the explicit results listed above have applied to the reduced matrix elements of composite operators at total momentum $p^{\prime}-p$ equal to zero, leaving the momentum squared of each external gauge field, $p^{2}$, and the mass $m$ of the fermions as independent parameters. Existing physical applications typically involve the reduced matrix elements in the limits in which $-p^{2}$ goes either to zero or to infinity with respect to $m^{2}$. It is well-known that at one loop the reduced matrix element of the current divergence vanishes as $-p^{2} / m^{2} \rightarrow 0$, whereas the pseudoscalar density $m j_{5}$ drops out in the opposite limit, see (4.5-6). Evidently neither of these 
observations depends on the precise normalization of the objects they apply to. Both statements remain true at two loops, the first, exhibited in (4.10), being obviously the non-trivial one. Such behavior has been observed in the formalism of [10] as well. The noncommutativity of the infrared limits has also been discussed at the two-loop level in a dispersive approach in [28]; however, the contention of that paper that different limits correspond to different currents is at odds with the point of view taken here.

For the abelian theory the vanishing to all orders of the reduced matrix element of $\partial_{\mu} j_{5}^{\mu}$ in the limit $-p^{2} / m^{2} \rightarrow 0$ is an aspect of the original Adler-Bardeen analysis [4]; the proof uses gauge invariance and analyticity. The background field method transfers at least the former tool to the nonabelian case. A putative generalized proof would start with the observation that if symmetry considerations are taken into account, the 1PI function of the (renormalized) current $j_{5}^{\lambda}$ and two gauge fields has the form [29], at ${p^{\prime 2}}^{2}=p^{2}$,

$$
\begin{gathered}
{\left[D_{1}\left(p^{2}\right)\left(\epsilon_{\mu \nu \alpha}^{\lambda} p^{\alpha}+\epsilon_{\mu \nu \alpha}^{\lambda} p^{\prime \alpha}\right)+D_{2}\left(p^{2}\right)\left(p_{\mu} \epsilon_{\nu \alpha \beta}^{\lambda} p^{\alpha} p^{\prime \beta}+p_{\nu}^{\prime} \epsilon_{\mu \alpha \beta}^{\lambda} p^{\alpha} p^{\prime \beta}\right)\right.} \\
\left.+D_{3}\left(p^{2}\right)\left(p_{\nu} \epsilon_{\mu \alpha \beta}^{\lambda} p^{\alpha} p^{\prime \beta}+p_{\mu}^{\prime} \epsilon_{\nu \alpha \beta}^{\lambda} p^{\alpha} p^{\prime \beta}\right)\right] \delta_{a b}
\end{gathered}
$$

and the (renormalized) reduced matrix element of $\partial_{\lambda} j_{5}^{\lambda}$ is $-2 i D_{1}$. Gauge invariance of the background field effective action requires (5.1) to vanish when contracted with $p^{\mu}$ or $p^{\prime \nu}$, implying $D_{1}\left(p^{2}\right)=-p^{2}\left(D_{2}\left(p^{2}\right)+D_{3}\left(p^{2}\right)\right)$. If $D_{2}$ and $D_{3}$ are sufficiently well-behaved at $p^{2}=0$ it follows that $D_{1}\left(p^{2}=0\right)=0$; but the premise is not at all obvious in the nonabelian case, whereas the $D$ 's are known to be analytic near $p^{2}=0$ in the abelian theory with massive fermions.

The interpolation between the two extremes $-p^{2} / m^{2} \rightarrow 0$ and $m^{2} /\left(-p^{2}\right) \rightarrow 0$ involves $m$ explicitly and is therefore highly scheme-dependent. At low orders, however, relatively simple results may emerge; for instance, for the two-loop $(1-\alpha)^{2}$ terms of $\hat{\Gamma}_{\partial_{\mu}\left[j_{5}^{\mu}\right]_{M S}}$ and $\hat{\Gamma}_{m\left[j_{5}\right]_{M S}}$ the interpolation turns out to exactly parallel that of the complete one-loop functions, as in (4.2) and (4.3).

Granted the distribution of nonzero results between the divergence of the current and the pseudoscalar density, what are those results taken by themselves? The simplest case is the massive abelian one at $p=p^{\prime}, p^{2} \rightarrow 0$, treated by Adler and Bardeen [4]. In that case the anomaly equation relates the matrix element 
of the canonical pseudoscalar density $m\left[j_{5}\right]_{C}$ to that of $g^{2}[F \tilde{F}]_{C}$; the latter is no longer sensitive to mixing because the matrix element of $\partial_{\mu} j_{5}^{\mu}$ vanishes under the circumstances. (In this sense what is often referred to in the literature as the AdlerBardeen current is merely an accessory to the Adler-Bardeen [4] theorem.) It can be shown by inspection of diagrams that the complete reduced matrix element of $g^{2}[F \tilde{F}]_{C}$, hence also that of $m\left[j_{5}\right]_{C}$, is given by its lowest order value, a constant times $g^{2}$. Each of these two reduced matrix elements satisfies a renormalization group equation

$$
\left[\mu \frac{\partial}{\partial \mu}+\beta \frac{\partial}{\partial g^{2}}+\gamma_{m} m^{2} \frac{\partial}{\partial m^{2}}+\beta_{\alpha} \frac{\partial}{\partial \alpha}-2 \gamma_{A}\right] \hat{\Gamma}_{\mathcal{O}}\left(p^{\prime}=p, p^{2}=0 ; m \neq 0\right)=0
$$

where the anomalous dimension of the photon is essentially identical to the betafunction, $\gamma_{A}=\beta / 2 g^{2}$; the exact results for the $\hat{\Gamma}_{\mathcal{O}}\left(p^{\prime}=p ; p^{2}=0 ; m \neq 0\right)$ thus satisfy the renormalization group equations (5.2) in the minimal nontrivial way [1], with $\mu \frac{\partial}{\partial \mu} \hat{\Gamma}_{\mathcal{O}}=0$. The explicit two-loop values, (4.19) and (4.22) at $c_{A}=0$, are obviously compatible with the general result.

Still in the abelian theory but with vanishing fermion mass, it becomes impossible to disentangle the divergence of the axial current and the antisymmetric photon tensor by the infrared behavior of their reduced matrix elements, because of mixing and the attendant normalization ambiguity. Perhaps the cleanest matrix element in this kinematical configuration is that of the divergence of the canonical axial current, $\partial_{\mu}\left[j_{5}^{\mu}\right]_{C}$, which satisfies a renormalization equation of the form (5.2). However, the vanishing anomalous dimension of $\left[j_{5}^{\mu}\right]_{C}$ now merely conceals the underlying non-trivial renormalization effects and the solution to (5.2) is no longer exhausted by a one-loop term. Explicitly, from (2.12), (2.11), (2.7), and (4.17) (with $c_{A}=0$ ), and using the lowest order value of $\beta$,

$$
\left.\hat{\Gamma}_{\partial_{\mu}\left[j_{5}^{\mu}\right]_{C}}\left(p^{\prime}=p ; m=0\right)\right|_{\text {abelian }}=-\frac{g^{2} n_{R} T_{R}}{2 \pi^{2}}-\frac{9 g^{4} c_{R} n_{R} T_{R}}{32 \pi^{4}}+\ldots
$$

The renormalization group calls for a logarithmic term at order $g^{6}$ to match the $g^{4}$ contribution; this term is supplied by three-loop diagrams as in Figure 8, computed and discussed in [26]. Of course higher powers of $\ln \frac{-p^{2}}{\mu^{2}}$ will emerge from the ellipsis if the calculation is pursued to higher orders. 
Turning now to the nonabelian case, consider only the case of massive fermions and $p^{\prime}=p, p^{2} \rightarrow 0$, obviously the simpler. In ordinary perturbation theory the analogy with the abelian situation is obscured from the onset, because the anomalous dimension of the gluon, $\gamma_{A}$, is then no longer simply related to the beta-function; this spoils the chances of a purely lowest order reduced matrix element, as this could no longer saturate the renormalization group equation. In the background field method, $\gamma_{A}$ is again equal to $\frac{1}{2} \frac{\beta}{g^{2}}$. As a result there can be no logarithms in the reduced matrix element of $m\left[j_{5}\right]_{C}$ or $g^{2}[F \tilde{F}]_{C}$ at the next-to-leading order, a fact borne out by the explicit results, (4.19) and (4.22) (in contrast to the corresponding quantities in [10], obtained in ordinary perturbation theory). Yet, as these equations show, even in the background field formalism $\hat{\Gamma}_{m\left[j_{5}\right]_{C}}\left(p^{\prime}=p, p^{2} \rightarrow 0 ; m \neq 0\right)$ and $\hat{\Gamma}_{g^{2}[F \tilde{F}]_{C}}\left(p^{\prime}=p, p^{2} \rightarrow 0 ; m \neq 0\right)$ have corrections to their leading orders, in contrast to the abelian case.

A salient feature of these corrections is their dependence on the gauge parameter $\alpha$. That an expression for general $p$ and $p^{\prime}$ such as (4.20) exhibits such dependence is not surprising, because there is no reason to expect gauge invariant results as long as the external gluons are off-shell. Of course infrared problems make it impossible to take $p^{2} \rightarrow 0$ or ${p^{\prime}}^{2} \rightarrow 0$ in (4.20). If $p^{\prime}$ is first taken to be equal to $p$ the one-loop result becomes independent of $p^{2}$ and $p^{2}$, see (4.22), and in particular finite as $p^{2} \rightarrow 0$, but this is deceptive; the vanishing of the anomalous dimension of $g^{2}[F \tilde{F}]_{C}$ is accommodated in $\hat{\Gamma}_{g^{2}[F \tilde{F}]_{C}}\left(p^{\prime}=p\right)$ by the occurrence of powers of $\ln \frac{-p^{2}}{\mu^{2}}$ starting at the two-loop level.

The noncommutativity of these limits is reminiscent of what happens with the divergence of the axial current at one loop. The analogy may be reinforced by introduction of a small finite gluon mass $M$. This was probably first considered by Chanowitz [30], who employed spontaneous symmetry breaking to procure a mass for the gauge fields. As long as gauge fields contribute through no more than one loop the mass may also be introduced straightforwardly, via a massive propagator

$$
-i\left[\frac{\eta_{\mu \nu}}{k^{2}-M^{2}}-\frac{(1-\alpha) k^{\mu} k^{\nu}}{\left(k^{2}-M^{2}\right)\left(k^{2}-\alpha M^{2}\right)}\right]
$$

Recalculation of the diagrams in Figure 2 with this propagator leads to a general- 
ization of (4.20). In the Feynman gauge,

$$
\left.\hat{\Gamma}_{[F \tilde{F}]_{M S}}\right|_{\text {one loop }}=-\frac{2 g^{2} c_{A}}{\pi^{2}}\left(1+\left(2 M^{2}-\frac{1}{2}\left(p-p^{\prime}\right)^{2}\right) I_{00}\left(p, p^{\prime} ; M\right)\right) .
$$

This should be compared with the expression for the one-loop reduced matrix element of $\partial_{\mu} j_{5}^{\mu}, 4.2$. At $p^{\prime}=p$, i.e. as the composite operator is integrated over spacetime, the two results are completely analogous. In particular, as long as $M$ is finite the one-loop correction to $\hat{\Gamma}_{[F \tilde{F}]_{M S}}$ disappears at $p^{\prime}=p$. This is actually also true for gauges $\alpha \neq 1$, though the expression for general $p$ and $p^{\prime}$ is not quite as simple as (5.5) above. The impossibility of a direct generalization of the Adler-Bardeen results to the nonabelian case appears therefore attendant upon the masslessness of the gauge fields.

We may reconsider similarly the calculation of $\hat{\Gamma}_{\left[j_{5}\right]_{M S}}\left(p=p^{\prime}, p^{2} \rightarrow 0 ; m \neq 0\right)$ by including a small gluon mass, taking the limit $\frac{M}{m} \rightarrow 0$. Not surprisingly, the entries in Table 3 that betray their sensitivity to the infrared behavior of the gauge fields by the presence of $\ln \left(-p^{2}\right)$ have to be changed to reflect the new circumstances. Diagrams I through VI, VIII and XI are not affected, but VII now yields

$$
c_{A}\left((-12-4 \alpha) \ln \frac{M^{2}}{m^{2}}-4 \alpha \ln \alpha+2+6 \alpha\right)
$$

and IX $+\mathrm{X}$

$$
2 c_{A}\left(\frac{4 \alpha}{\epsilon}-4 \alpha L_{m}+(6+2 \alpha) \ln \frac{M^{2}}{m^{2}}+2 \alpha \ln \alpha-2-4 \alpha\right) .
$$

Compared with table 3 as it stands the extra contributions add up to $-16 c_{A}[1+$ $\left.\frac{3}{4}(1-\alpha)-\frac{1}{8}(1-\alpha)^{2}\right]$, which is precisely what is needed to eliminate the terms proportional to $c_{A} g^{4}$.

The existence of a finite one-loop contribution to the reduced matrix element of $g^{2}[F \tilde{F}]_{C}$ at $p^{\prime}=p$ appears to be at odds with a semiclassical interpretation of $\frac{1}{64 \pi^{2}} g^{2}[F \tilde{F}]_{C}$ as the Pontryagin density, the integral of which is the instanton number. It is tempting to try to remove the extra factor by a finite renormalization, but that would interfere with the vanishing of the anomalous dimension of $g^{2}[F \tilde{F}]_{C}$ and leave explicit logarithms untouched. However, the sensitivity of the one-loop reduced matrix element to the infrared arrangement of the theory suggests that 
the problem is an infrared artifact of perturbation theory, and that its vanishing at infinitesimal gluon mass mimicks behavior induced by a nonperturbative physical infrared cutoff. (This appears to be also the point of view advocated in [15]; the alternative point of view that all or part of the one-loop matrix element of $g^{2} F \tilde{F}$ is a signature of a real effect involving the vacuum angle $\theta_{Q C D}$ [31] is developed in [13, 14].)

\section{Conclusion}

Since its identification more than twenty years ago, the axial anomaly has never been far from the spotlight of theoretical physics. Regarding most of its aspects it could therefore be plausibly argued that no relevant insight remains to be put forward. Yet the continuing flow of papers related to the interplay of the anomaly and the process of renormalization bears witness that the concrete organization of the currently available intuition in specific physical contexts is still not without challenges. The rationale of the present paper was the consideration that for nonabelian gauge theory and the practically important formalism of dimensional regularization, a truly comprehensive discussion was lacking in the literature.

A review was given of the renormalization group arguments that lead to a convenient normalization of the singlet axial current and the operators related to its divergence. These considerations were matched to a derivation of the anomaly equation using dimensional regularization with what is probably the most straightforward treatment of pseudotensorial objects, that according to 't Hooft and Veltman. This settled the issue in principle. To illustrate how this works in practice, and verify that the abstract argument was sound, I computed the two-gluon matrix element of each term in the anomaly equation through two loops, including features neglected in previous treatments. Such calculations supply, apart from the desired reassurance, matrix elements that may be of independent interest, if not of immediate physical significance. 


\section{Acknowledgements}

This work was supported in part by the National Science Foundation under grant NSF/PHY-89-15286. I have benefited from discussions with members of the UCLA Theoretical Physics Group, particularly Brian Hill, and with Stefano Forte. 


\section{References}

[1] J. Bell and R. Jackiw, Nuovo Cim. 60A, 47 (1969); S. Adler, Phys. Rev. 177, 2426.

[2] H. Fukuda and Y. Miyamoto, Prog. Theor. Phys. 4, 347 (1949); J. Steinberger, Phys Rev. 76, 1180 (1949); J. Schwinger, Phys. Rev. 82, 664 (1951); K. Johnson, Phys. Lett. 5, 253 (1963).

[3] S. Treiman, R. Jackiw, B. Zumino, and E. Witten, Current Algebra and Anomalies (Princeton University Press, Princeton, 1985).

[4] S. Adler and W. Bardeen, Phys. Rev. 182, 1517 (1969); S. Adler, in Lectures on Elementary Particles and Quantum Field Theory, ed. S. Deser, M. Grisaru and H. Pendleton (MIT Press, Cambridge Mass. 1970).

[5] M. Grisaru, in Recent Developments in Gravitation, ed. M. Levy and S. Deser (Plenum Press, New York, 1979).

[6] G. Altarelli, in The Challenging Questions, Proc. of the 1989 Erice Summer School, ed. A. Zichichi (Plenum, New York, 1990).

[7] M. Grisaru, B. Milewski and D. Zanon, Nucl. Phys. B266, 589 (1986).

[8] G. 't Hooft and M. Veltman, Nucl. Phys. B44, 189 (1972).

[9] D.R.T. Jones and J.P. Leveille, Nucl. Phys. B206, 473 (1982).

[10] R. Akhoury and S. Titard, Michigan/Southhampton preprint UM-TH-91-21, SHEP-91/92-2 (1991).

[11] A. Zee, Phys. Rev. Lett. 29, 1198 (1972).

[12] S.-Y. Pi and S.-S. Shei, Phys. Rev. D11, 2946 (1975).

[13] M.A. Shifman and A.I. Vainshtein, Nucl. Phys. B277, 456 (1986).

[14] M.A. Shifman and A.I. Vainshtein, Nucl. Phys. B365, 312 (1991).

[15] A. Jogansen, Sov. J. Nucl. Phys. 54, 349 (1991).

[16] W. Bardeen, Nucl. Phys. B75, 246 (1974).

[17] P. Breitenlohner, D. Maison and K. Stelle, Phys. Lett. 134B, 63 (1984).

[18] C. Lucchesi, O. Piguet and K. Sibold, Int. J. Mod. Phys. A2, 385 (1987).

[19] R. Crewther, in Facts and Prospects of Gauge Theories, Acta Phys. Austr. Suppl. XIX, 47 (1978).

[20] J. Collins, Renormalization (Cambridge University Press, Cambridge U.K., 1984).

[21] P. Breitenlohner and D. Maison, Comm. Math. Phys. 52, 11 (1977).

[22] M. Bos, Phys. Lett. 189B, 435 (1987); Ann. Phys. (NY) 181, 177 (1988).

[23] C. Schubert, Nucl. Phys. B323, 478 (1989).

[24] L. Abbott, Nucl. Phys. B185, 189 (1981). 
[25] G. Gabadadze and A. Pivovarov, JETP Letters 54, 298 (1991).

[26] A.A. Anselm and A.A. Jogansen, Sov. Phys. JETP 69, 670 (1989).

[27] G. 't Hooft, Nucl. Phys. B61, 455 (1973).

[28] V. Zakharov, Phys. Rev. D42, 1208 (1990).

[29] L. Rosenberg, Phys. Rev. 129, 2786 (1963).

[30] M. Chanowitz, Phys. Rev. D9, 503 (1974).

[31] R. Jackiw and C. Rebbi, Phys. Rev. Lett. 37, 172 (1976); C. Callan, R. Dashen and D. Gross, Phys. Lett. 63B, 334 (1976). 


\section{Table Captions}

Table 1. Two-loop contributions to the unrenormalized reduced matrix element of $\partial_{\mu} j_{5}^{\mu}$ at $p^{\prime}=p$ and $m=0$, numbered as in Fig. 5. A common factor $g^{4} n_{R} T_{R} /(4 \pi)^{4}$ is omitted.

Table 2. Two-loop contributions to the unrenormalized reduced matrix element of $\partial_{\mu} j_{5}^{\mu}$ for $p^{\prime}=p, p^{2} \rightarrow 0$ and $m \neq 0$, numbered as in Fig. 5. A common factor $g^{4} n_{R} T_{R} /(4 \pi)^{4}$ is omitted.

Table 3. Two-loop contributions to the unrenormalized reduced matrix element of $j_{5}$ for $p^{\prime}=p, p^{2} \rightarrow 0$ and $m \neq 0$, numbered as in Fig. 5. A common factor $g^{4} n_{R} T_{R} /(4 \pi)^{4} m$ is omitted.

Table 4. Pole parts of the two-loop Feynman gauge contributions to the unrenormalized reduced matrix element of $F \tilde{F}$ for $p^{\prime}=p$, numbered as in Fig. 7. A common factor $g^{4} /(4 \pi)^{4}$ is omitted, as are all terms of the form $\frac{1}{\epsilon} L_{p}$. In XXXI, $\left(^{*}\right)$ marks the result for $m=0, p^{2} \neq 0,(* *)$ that of $m \neq 0, p^{2} \rightarrow 0$.

\section{Figure Captions}

Fig. 1. One-loop diagram relevant for the renormalization of $j_{5}^{\mu}$ and $j_{5}$.

Fig. 2. One loop diagrams contributing to the two-gluon 1 PI function of $F \tilde{F}$.

Fig. 3. One-loop diagram leading to mixing of $\partial_{\mu} j_{5}^{\mu}$ with $F \tilde{F}$.

Fig. 4. One-loop diagram for the two-gluon $1 \mathrm{PI}$ functions of $\partial_{\mu} j_{5}^{\mu}, j_{5}$, or $\mathcal{O}_{a n}$.

Fig. 5. Two-loop diagrams contributing to the two-gluon $1 \mathrm{PI}$ functions of $\partial_{\mu} j_{5}^{\mu}$ and $j_{5}$.

Fig. 6. One-loop diagrams contributing to the $\psi-\bar{\psi} 1 \mathrm{PI}$ function of $\mathcal{O}_{a n}$.

Fig. 7. Two-loop diagrams contributing to the two-gluon 1PI function of $F \tilde{F}$. For each ghost or quark line both orientations should be taken into account.

Fig. 8. A three-loop contribution to the two-gluon 1PI function of $\partial_{\mu} j_{5}^{\mu}$. 
TABLE 1

\begin{tabular}{lccc} 
& $(1-\alpha)^{0}$ & $(1-\alpha)^{1}$ & $(1-\alpha)^{2}$ \\
\hline $\mathbf{I}+\mathbf{I I}$ & $2 \mathrm{c}_{\mathrm{R}}\left(\frac{12}{\epsilon}-12 \mathrm{~L}_{p}+21\right)$ & $2 \mathrm{c}_{\mathrm{R}}\left(-\frac{12}{\epsilon}+12 \mathrm{~L}_{p}-21\right)$ & 0 \\
$\mathbf{I I I}$ & $\mathrm{c}_{\mathrm{R}}\left(\frac{8}{\epsilon}-8 \mathrm{~L}_{p}+14\right)$ & $\mathrm{c}_{\mathrm{R}}\left(-\frac{8}{\epsilon}+8 \mathrm{~L}_{p}-14\right)$ & 0 \\
$\mathbf{I V + V}$ & $2\left(\mathrm{c}_{\mathrm{R}}-\frac{1}{2} \mathrm{c}_{\mathrm{A}}\right)\left(-\frac{8}{\epsilon}+8 \mathrm{~L}_{p}-18\right)$ & $2\left(\mathrm{c}_{\mathrm{R}}-\frac{1}{2} \mathrm{c}_{\mathrm{A}}\right)\left(\frac{8}{\epsilon}-8 \mathrm{~L}_{p}+14\right)$ & 0 \\
$\mathbf{V I}$ & $\mathrm{c}_{\mathrm{R}}\left(-\frac{16}{\epsilon}+16 \mathrm{~L}_{p}-52\right)$ & $\mathrm{c}_{\mathrm{R}}\left(\frac{16}{\epsilon}-16 \mathrm{~L}_{p}+28\right)$ & 0 \\
$\mathbf{V I I}$ & $\mathrm{c}_{\mathrm{A}}\left(-\frac{32}{\epsilon}+32 \mathrm{~L}_{p}-104\right)$ & $\mathrm{c}_{\mathrm{A}}\left(\frac{8}{\epsilon}-8 \mathrm{~L}_{p}+10\right)$ & 0 \\
$\mathbf{V I I I}$ & 0 & 0 & 0 \\
$\mathbf{I X}+\mathbf{X}$ & $2 \mathrm{c}_{\mathrm{A}}\left(\frac{4}{\epsilon}-4 \mathrm{~L}_{p}+\frac{31}{3}+8 \zeta(3)\right)$ & $2 \mathrm{c}_{\mathrm{A}}\left(\frac{2}{\epsilon}-2 \mathrm{~L}_{p}-\frac{9}{2}-4 \zeta(3)\right)$ & $4 \mathrm{c}_{\mathrm{A}}$ \\
$\mathbf{X I}$ & $\mathrm{c}_{\mathrm{A}}\left(\frac{16}{\epsilon}-16 \mathrm{~L}_{p}+\frac{100}{3}-16 \zeta(3)\right)$ & $\mathrm{c}_{\mathrm{A}}\left(-\frac{4}{\epsilon}+4 \mathrm{~L}_{p}-11+8 \zeta(3)\right)$ & 0 \\
\hline total & $-32 \mathrm{c}_{\mathrm{R}}-32 \mathrm{c}_{\mathrm{A}}$ & $-24 \mathrm{c}_{\mathrm{A}}$ & $4 \mathrm{c}_{\mathrm{A}}$
\end{tabular}




\section{TABLE 2}

\begin{tabular}{|c|c|c|c|}
\hline & $(1-\alpha)^{0}$ & $(1-\alpha)^{1}$ & $(1-\alpha)^{2}$ \\
\hline $\mathbf{I}+\mathbf{I I}$ & $2 \mathrm{c}_{\mathrm{R}}\left(\frac{4}{\epsilon}-4 \mathrm{~L}_{m}-\frac{11}{3}\right)$ & $2 \mathrm{c}_{\mathrm{R}}\left(\frac{4}{\epsilon}-4 \mathrm{~L}_{m}-3\right)$ & 0 \\
\hline III & $\mathrm{c}_{\mathrm{R}}\left(-\frac{24}{\epsilon}+24 \mathrm{~L}_{m}+\frac{10}{3}\right)$ & $\mathrm{c}_{\mathrm{R}}\left(\frac{8}{\epsilon}-8 \mathrm{~L}_{m}-2\right)$ & 0 \\
\hline $\mathbf{I V}+\mathbf{V}$ & $2\left(\mathrm{c}_{\mathrm{R}}-\frac{1}{2} \mathrm{c}_{\mathrm{A}}\right)\left(\frac{8}{\epsilon}-8 \mathrm{~L}_{m}+2\right)$ & $2\left(\mathrm{c}_{\mathrm{R}}-\frac{1}{2} \mathrm{c}_{\mathrm{A}}\right)\left(-\frac{8}{\epsilon}+8 \mathrm{~L}_{m}+2\right)$ & 0 \\
\hline VI & 0 & $4 c_{R}$ & 0 \\
\hline VII & $\mathrm{c}_{\mathrm{A}}\left(-\frac{32}{\epsilon}+32 \mathrm{~L}_{m}+8\right)$ & $\mathrm{c}_{\mathrm{A}}\left(\frac{8}{\epsilon}-8 \mathrm{~L}_{m}-2\right)$ & 0 \\
\hline VIII & 0 & 0 & 0 \\
\hline $\mathbf{I X}+\mathbf{X}$ & $2 \mathrm{c}_{\mathrm{A}}\left(\frac{12}{\epsilon}-12 \mathrm{~L}_{m}-1\right)$ & $2 \mathrm{c}_{\mathrm{A}}\left(-\frac{6}{\epsilon}+6 \mathrm{~L}_{m}+\frac{5}{2}\right)$ & 0 \\
\hline XI & $\mathrm{c}_{\mathrm{A}}\left(\frac{16}{\epsilon}-16 \mathrm{~L}_{m}-4\right)$ & $\mathrm{c}_{\mathrm{A}}\left(-\frac{4}{\epsilon}+4 \mathrm{~L}_{m}-1\right)$ & 0 \\
\hline total & 0 & 0 & 0 \\
\hline
\end{tabular}




\section{TABLE 3}

\begin{tabular}{lccc} 
& $(1-\alpha)^{0}$ & $(1-\alpha)^{1}$ & $(1-\alpha)^{2}$ \\
\hline $\mathbf{I}+\mathbf{I I}$ & $2 \mathrm{c}_{\mathrm{R}}\left(\frac{-16}{\epsilon}+16 \mathrm{~L}_{m}-\frac{16}{3}\right)$ & $2 \mathrm{c}_{\mathrm{R}}\left(\frac{8}{\epsilon}-8 \mathrm{~L}_{m}-2\right)$ & 0 \\
$\mathbf{I I I}$ & $\mathrm{c}_{\mathrm{R}}\left(-\frac{16}{\epsilon}+16 \mathrm{~L}_{m}-\frac{16}{3}\right)$ & $\mathrm{c}_{\mathrm{R}}\left(\frac{8}{\epsilon}-8 \mathrm{~L}_{m}-2\right)$ & 0 \\
$\mathbf{I V + V}$ & $2\left(\mathrm{c}_{\mathrm{R}}-\frac{1}{2} \mathrm{c}_{\mathrm{A}}\right)\left(\frac{8}{\epsilon}-8 \mathrm{~L}_{m}+2\right)$ & $2\left(\mathrm{c}_{\mathrm{R}}-\frac{1}{2} \mathrm{c}_{\mathrm{A}}\right)\left(-\frac{8}{\epsilon}+8 \mathrm{~L}_{m}+2\right)$ & 0 \\
$\mathbf{V I}$ & $\mathrm{c}_{\mathrm{R}}\left(\frac{32}{\epsilon}-32 \mathrm{~L}_{m}+20\right)$ & $\mathrm{c}_{\mathrm{R}}\left(-\frac{8}{\epsilon}+8 \mathrm{~L}_{m}+2\right)$ & 0 \\
$\mathbf{V I I}$ & $\mathrm{c}_{\mathrm{A}}\left(-16 \mathrm{~L}_{p}+16 \mathrm{~L}_{m}+56\right)$ & $\mathrm{c}_{\mathrm{A}}\left(4 \mathrm{~L}_{p}-4 \mathrm{~L}_{m}-6\right)$ & 0 \\
$\mathbf{V I I I}$ & 0 & 0 & $-2 \mathrm{c}_{\mathrm{A}}$ \\
$\mathbf{I X}+\mathbf{X}$ & $2 \mathrm{c}_{\mathrm{A}}\left(\frac{4}{\epsilon}+8 \mathrm{~L}_{p}-12 \mathrm{~L}_{m}-22\right)$ & $2 \mathrm{c}_{\mathrm{A}}\left(-\frac{4}{\epsilon}-2 \mathrm{~L}_{p}+6 \mathrm{~L}_{m}+10\right)$ & 0 \\
$\mathbf{X I}$ & $6 \mathrm{c}_{\mathrm{A}}$ & 0 & $-2 \mathrm{c}_{\mathrm{A}}$
\end{tabular}


TABLE 4

\begin{tabular}{|c|c|c|c|}
\hline I & $\left(-\frac{148}{\epsilon^{2}}-\frac{541}{\epsilon}\right) \mathrm{c}_{\mathrm{A}}^{2}$ & XIX & 0 \\
\hline II & $\left(-\frac{320}{\epsilon^{2}}-\frac{1104}{\epsilon}\right) \mathrm{c}_{\mathrm{A}}^{2}$ & $\mathrm{XX}$ & $\frac{48}{\epsilon} c_{\mathrm{A}}^{2}$ \\
\hline III & $\left(\frac{16}{\epsilon^{2}}+\frac{2}{\epsilon}\right) \mathrm{c}_{\mathrm{A}}^{2}$ & XXI & $\left(\frac{192}{\epsilon^{2}}+\frac{624}{\epsilon}\right) c_{\mathrm{A}}^{2}$ \\
\hline IV & $\left(\frac{12}{\epsilon^{2}}+\frac{35}{\epsilon}\right) \mathrm{c}_{\mathrm{A}}^{2}$ & XXII & $\left(-\frac{192}{\epsilon^{2}}-\frac{480}{\epsilon}\right) c_{\mathrm{A}}^{2}$ \\
\hline V & 0 & XXIII & $\left(\frac{108}{\epsilon^{2}}+\frac{255}{\epsilon}\right) \mathrm{c}_{\mathrm{A}}^{2}$ \\
\hline VI & $\left(-\frac{2}{\epsilon}\right) \mathrm{c}_{\mathrm{A}}^{2}$ & XXIV & $\left(-\frac{4}{\epsilon^{2}}-\frac{9}{\epsilon}\right) \mathrm{c}_{\mathrm{A}}^{2}$ \\
\hline VII & $\left(-\frac{608}{3 \epsilon^{2}}-\frac{5960}{9 \epsilon}\right) c_{\mathrm{A}}^{2}$ & XXV & $\left(\frac{144}{\epsilon^{2}}+\frac{956}{3 \epsilon}\right) c_{\mathrm{A}}^{2}$ \\
\hline VIII & $\left(-\frac{304}{3 \epsilon^{2}}-\frac{2716}{9 \epsilon}\right) \mathrm{c}_{\mathrm{A}}^{2}$ & XXVI & $\left(\frac{16}{\epsilon^{2}}+\frac{140}{3 \epsilon}\right) \mathrm{c}_{\mathrm{A}}^{2}$ \\
\hline IX & $\left(-\frac{32}{3 \epsilon^{2}}-\frac{344}{9 \epsilon}\right) \mathrm{c}_{\mathrm{A}}^{2}$ & XXVII & $\left(-\frac{192}{\epsilon^{2}}-\frac{384}{\epsilon}\right) \mathrm{c}_{\mathrm{A}}^{2}$ \\
\hline $\mathbf{X}$ & $\left(-\frac{16}{3 \epsilon^{2}}-\frac{196}{9 \epsilon}\right) c_{\AA}^{2}$ & XXVIII & $\left(-\frac{36}{\epsilon^{2}}-\frac{81}{\epsilon}\right) c_{\mathrm{A}}^{2}$ \\
\hline XI & $-\frac{18}{\epsilon} c_{\mathrm{A}}^{2}$ & XXIX & $\left(-\frac{4}{\epsilon^{2}}-\frac{9}{\epsilon}\right) \mathrm{c}_{\mathrm{A}}^{2}$ \\
\hline XII & $\frac{2}{\epsilon} c_{\mathrm{A}}^{2}$ & $\mathbf{X X X}$ & $\left(-\frac{192}{\epsilon^{2}}-\frac{432}{\epsilon}\right) \mathrm{c}_{\mathrm{A}} \mathrm{n}_{\mathrm{R}} \mathrm{T}_{\mathrm{R}}$ \\
\hline XIII & $\left(\frac{144}{\epsilon^{2}}+\frac{396}{\epsilon}\right) \mathrm{c}_{\mathrm{A}}^{2}$ & XXXI & $\frac{128}{\epsilon} c_{\mathrm{R}} \mathrm{n}_{\mathrm{R}} \mathrm{T}_{\mathrm{R}}(*),-\frac{64}{\epsilon} \mathrm{c}_{\mathrm{R}} \mathrm{n}_{\mathrm{R}} \mathrm{T}_{\mathrm{R}}(* *)$ \\
\hline XIV & 0 & XXXII & $\frac{64}{\epsilon}\left(\mathrm{c}_{\mathrm{R}}-\frac{1}{2} \mathrm{c}_{\mathrm{A}}\right) \mathrm{n}_{\mathrm{R}} \mathrm{T}_{\mathrm{R}}$ \\
\hline XV & $\left(\frac{108}{\epsilon^{2}}+\frac{243}{\epsilon}\right) \mathrm{c}_{\mathrm{A}}^{2}$ & XXXIII & $\left(\frac{512}{3 \epsilon^{2}}+\frac{4736}{9 \epsilon}\right) \mathrm{c}_{\mathrm{A}} \mathrm{n}_{\mathrm{R}} \mathrm{T}_{\mathrm{R}}$ \\
\hline XVI & $\left(-\frac{4}{\epsilon^{2}}-\frac{1}{\epsilon}\right) \mathrm{c}_{\mathrm{A}}^{2}$ & XXXIV & $\left(\frac{256}{3 \epsilon^{2}}+\frac{2176}{9 \epsilon}\right) \mathrm{c}_{\mathrm{A}} \mathrm{n}_{\mathrm{R}} \mathrm{T}_{\mathrm{R}}$ \\
\hline XVII & $\left(\frac{192}{\epsilon^{2}}+\frac{576}{\epsilon}\right) \mathrm{c}_{\mathrm{A}}^{2}$ & XXXV & $\left(\frac{64}{\epsilon^{2}}+\frac{112}{\epsilon}\right) \mathrm{c}_{\mathrm{A}} \mathrm{n}_{\mathrm{R}} \mathrm{T}_{\mathrm{R}}$ \\
\hline XVIII & $\left(\frac{288}{\epsilon^{2}}+\frac{792}{\epsilon}\right) \mathrm{c}_{\mathrm{A}}^{2}$ & XXXVI & $\left(-\frac{128}{\epsilon^{2}}-\frac{800}{3 \epsilon}\right) \mathrm{c}_{\mathrm{A}} \mathrm{n}_{\mathrm{R}} \mathrm{T}_{\mathrm{R}}$ \\
\hline
\end{tabular}

